\title{
A VOLTERRA TYPE DERIVATIVE OF THE LEBESGUE INTEGRAL
}

\author{
WASHEK F. PFEFFER
}

(Communicated by Andrew M. Bruckner)

\begin{abstract}
Using functions of bounded variation, we define a Volterra type derivative of the linear functional associated with a Lebesgue integrable function and show that it is equal to this function almost everywhere.
\end{abstract}

If $\Omega$ is an open subset of $\mathbf{R}^{m}$ and $f \in L_{\text {loc }}^{1}(\Omega)$, let

$$
F(\theta)=\int_{\Omega} f \theta
$$

for each $\theta \in L_{\circ}^{\infty}(\Omega)$, where $L_{\circ}^{\infty}(\Omega)$ is the linear space of all $\theta \in L^{\infty}(\Omega)$ that vanish almost everywhere outside a compact subset of $\Omega$. It was shown in [P1, Corollary] that the Volterra derivative $\delta F / \delta \theta$ with respect to continuous functions $\theta$ displays a rather special behavior: it is continuous on the subset of $\Omega$ where it exists (cf. also [HN, Theorem 2]). In this note we show that using functions $\theta$ of bounded variation, a more natural type of the Volterra derivative $\mathfrak{D} F$ of $F$ can be defined. By controling a Vitali type regularity of the functions with respect to which we differentiate, we achieve that $\mathfrak{D} F=f$ almost everywhere in $\Omega$ whenever $f \in L_{\text {loc }}^{\infty}(\Omega)$. This result is used in [KMP] to show that a certain conditionally convergent integral in $\mathbf{R}^{m}$ is invariant with respect to lipeomorphisms.

Throughout, we fix an integer $m \geq 1$ and an open set $\Omega \subset \mathbf{R}^{m}$. The norms in $L^{1}(\Omega)$ and $L^{\infty}(\Omega)$ are denoted by $|\cdot|_{1}$ and $|\cdot|_{\infty}$, respectively. Unless stated otherwise, all functions and functionals we consider are real valued.

For $x=\left(\xi_{1}, \ldots, \xi_{m}\right)$ in $\mathbf{R}^{m}$ and $\varepsilon>0$, let $|x|=\sqrt{\xi_{1}^{2}+\cdots+\xi_{m}^{2}}$ and $U(x, \varepsilon)=\left\{y \in \mathbf{R}^{m}:|x-y|<\varepsilon\right\}$. If $E \subset \mathbf{R}^{m}$ then $E^{-}, d(E)$, and $|E|$ denote the closure, diameter, and the outer Lebesgue measure of $E$, respectively. Given a nonnegative function $\theta$ defined on $\Omega$ and $\alpha \geq 0$, we set $S_{\theta}(\alpha)=\{x \in \Omega: \theta(x)>\alpha\}$; we write $S_{\theta}$ instead of $S_{\theta}(0)$ and let $d(\theta)=d\left(S_{\theta}\right)$. We say that a function $\theta \in L^{1}(\Omega)$ is normalized whenever $|\theta|_{1}=\left|S_{\theta}\right|$.

Received by the editors June 7, 1991; presented at the 15th Summer Symposium in Real Analysis, Czechoslovakia, August 1991.

1991 Mathematics Subject Classification. Primary 28A15; Secondary 46G05. 
Following [G, Definition 1.1], we say that $\theta \in L^{1}(\Omega)$ is of bounded variation if its distributional gradient $D \theta$ is a vector-valued Borel measure in $\Omega$ whose variation $|D \theta|$ is finite; we set $\|\theta\|=|D \theta|(\Omega)$. The family of all nonnegative functions $\theta \in L_{\circ}^{\infty}(\Omega)$ that are of bounded variation is denoted by $B V_{+}$. The regularity of $\theta \in B V_{+}$is the number

$$
r(\theta)= \begin{cases}|\theta|_{1} /[d(\theta)\|\theta\|] & \text { if } d(\theta)\|\theta\|>0 \\ 0 & \text { otherwise. }\end{cases}
$$

The following lemma relates $r(\theta)$ to the usual concept of regularity of sets defined in [S, Chapter 4, §2].

Lemma 1. There is a constant $c>0$, depending only on the dimension $m$, such that

$$
\int_{S_{\theta}(\alpha)} \theta \leq c\left|S_{\theta}(\alpha)\right|^{1 / m}\|\theta\|
$$

for each $\theta \in B V_{+}$and $\alpha \geq 0$. In particular,

$$
\frac{r(\theta)}{c} \leq \frac{\left|S_{\theta}\right|^{1 / m}}{d\left(S_{\theta}\right)} \quad \text { and } \quad \int_{S_{\theta}(\alpha)} \theta \leq \frac{c}{\sqrt[m]{\alpha}} \cdot \frac{\left|S_{\theta}\right|}{r(\theta)},
$$

where the first inequality holds for each $\theta \in B V_{+}$and the second for each normalized $\theta \in B V_{+}$and $\alpha>0$.

Proof. If $m \geq 2$ the Hölder and Sobolev inequalities yield

$$
\int_{S_{\theta}(\alpha)} \theta \leq\left|S_{\theta}(\alpha)\right|^{1 / m}\left(\int_{S_{\theta}} \theta^{m /(m-1)}\right)^{(m-1) / m} \leq c_{m}\left|S_{\theta}(\alpha)\right|^{1 / m}\|\theta\|
$$

where $c_{m}$ is a positive constant depending only on $m$ (see [G, Theorem 1.28]). If $m=1$ then

$$
\int_{S_{\theta}(\alpha)} \theta \leq\left|S_{\theta}(\alpha)\right| \cdot|\theta|_{\infty} \leq\left|S_{\theta}(\alpha)\right| \cdot\|\theta\|
$$

Thus letting $c_{1}=1$ establishes the first inequality. The second inequality follows immediately from the first with $\alpha=0$. If $\theta$ is normalized then

$$
\alpha\left|S_{\theta}(\alpha)\right| \leq \int_{S_{\theta}(\alpha)} \theta \leq|\theta|_{1}=\left|S_{\theta}\right| \leq[d(\theta)]^{m} .
$$

For $\alpha>0$, the proof is completed by combining the first and last inequalities.

Definition. We say that a functional $J$ on $L_{\circ}^{\infty}(\Omega)$ is differentiable at $x \in \Omega$ if there is a real number $\alpha$ such that given $\varepsilon>0$ we can find a $\delta>0$ so that

$$
\left.\left.|J(\theta)-\alpha| \theta\right|_{1}|<\varepsilon| \theta\right|_{1}
$$

for each $\theta \in B V_{+}$with $x \in S_{\theta}^{-}, d(\theta)<\delta$, and $r(\theta)>\varepsilon$. If the inequality holds only when $\theta$ is, in addition, normalized and $|\theta|_{\infty}<1 / \varepsilon$, we say that $J$ is weakly differentiable at $x$.

The number $\alpha$ from the definition is uniquely determined, and we denote it by $\mathfrak{D} J(x)$ or $\mathfrak{D}_{w} J(x)$ according to whether $J$ is differentiable or weakly differentiable at $x$, respectively. If $J$ is differentiabe at $x$ it is also weakly differentiable at $x$, and $\mathfrak{D}_{w} J(x)=\mathfrak{D} J(x)$. The example below shows, however, that the converse is false for $m>1$. If $m=1$, the two concepts of differentiability coincide since $|\theta|_{\infty} \leq\|\theta\| \leq 1 / r(\theta)$ for each normalized $\theta \in B V_{+}$. 
Proposition. Let if $\in L_{\mathrm{loc}}^{1}(\Omega)$ and $F(\theta)=\int_{\Omega} f \theta$ for each $\theta \in L_{\circ}^{\infty}(\Omega)$. Suppose that $F$ is weakly differentiable at $x \in \Omega$ and $\mathfrak{D}_{w} F(x)=f(x)$. If $f$ is essentially bounded in a neighborhood of $x$, then $F$ is differentiable at $x$ and $\mathfrak{D} F(x)=f(x)$.

Proof. Choose $\delta>0$ so that $\alpha=\sup \operatorname{ess}_{y \in U(x, \delta)}|f(y)|$ is finite. Proceeding towards a contradiction, assume there is an $\varepsilon>0$ and normalized functions $\theta_{k} \in B V_{+}, k=1,2, \ldots$, such that $x \in S_{\theta_{k}}^{-}, d\left(\theta_{k}\right)<\delta / k, r\left(\theta_{k}\right)>\varepsilon$, and

$$
\left|\int_{\Omega} f \theta_{k}-f(x) \int_{\Omega} \theta_{k}\right|>\varepsilon \int_{\Omega} \theta_{k} .
$$

For $k, n=1,2, \ldots$, let $\beta_{k, n}=\left|S_{\theta_{k}}\right| /\left|\min \left\{\theta_{k}, n\right\}\right|_{1}$ and $\theta_{k, n}=$ $\beta_{k, n} \min \left\{\theta_{k}, n\right\}$. Each $\theta_{k, n}$ is a normalized function from $B V_{+}$and $\left\|\theta_{k, n}\right\| \leq$ $\beta_{k, n}\left\|\theta_{k}\right\|$. By Lemma 1, there is a constant $c>0$, depending only on $m$, such that

$$
\left|S_{\theta_{k}}\right| \geq \int_{\Omega} \min \left\{\theta_{k}, n\right\}=\int_{\Omega} \theta_{k}-\int_{S_{\theta_{k}}(n)} \theta_{k} \geq\left|S_{\theta_{k}}\right|\left(1-\frac{c}{\varepsilon} \cdot \frac{1}{\sqrt[m]{n}}\right)
$$

and

$$
\begin{aligned}
\left|\int_{\Omega} f \theta_{k}-\int_{\Omega} f \theta_{k, n}\right| & \leq \alpha\left[\int_{\Omega}\left|\theta_{k}-\beta_{k, n} \theta_{k}\right|+\int_{\Omega}\left|\beta_{k, n} \theta_{k}-\theta_{k, n}\right|\right] \\
& =\alpha\left[\left(1-\beta_{k, n}\right) \int_{\Omega} \theta_{k}+\beta_{k, n} \int_{\Omega}\left(\theta_{k}-\min \left\{\theta_{k}, n\right\}\right)\right] \\
& =\alpha\left[\left(1-\beta_{k, n}\right)\left|S_{\theta_{k}}\right|+\beta_{k, n} \int_{S_{\theta_{k}(n)}} \theta_{k}\right] \\
& \leq \alpha\left|S_{\theta_{k}}\right|\left(1-\beta_{k, n}+\beta_{k, n} \frac{c}{\varepsilon} \cdot \frac{1}{\sqrt[m]{n}}\right) .
\end{aligned}
$$

The first inequality yields

$$
1 \geq \frac{1}{\beta_{k, n}} \geq 1-\frac{c}{\varepsilon} \cdot \frac{1}{\sqrt[m]{n}}
$$

and hence $\lim _{n \rightarrow \infty} \beta_{k, n}=1$ uniformly in $k$. From this and the second inequality we see that

$$
\lim _{n \rightarrow \infty} \frac{1}{\left|S_{\theta_{k}}\right|} \int_{\Omega} f \theta_{k, n}=\frac{1}{\left|S_{\theta_{k}}\right|} \int_{\Omega} f \theta_{k}
$$

uniformly in $k$. Thus there is an integer $p \geq 1$ such that $\beta_{k, p}<2$ and

$$
\left|\frac{1}{\left|S_{\theta_{k}}\right|} \int_{\Omega} f \theta_{k, p}-f(x)\right|>\frac{\varepsilon}{2}
$$

for $k=1,2, \ldots$. Since $S_{\theta_{k}}=S_{\theta_{k, p}}$, we have $\left|S_{\theta_{k}}\right|=\int_{\Omega} \theta_{k, p}$ and

$$
\left|\int_{\Omega} f \theta_{k, p}-f(x) \int_{\Omega} \theta_{k, p}\right|>\frac{\varepsilon}{2} \int_{\Omega} \theta_{k, p} .
$$

As $\lim _{k \rightarrow \infty} d\left(\theta_{k, p}\right)=0, r\left(\theta_{k, p}\right)>\varepsilon / 2$, and $\left|\theta_{k, p}\right|_{\infty} \leq 2 p$, a contradiction follows. 
Lemma 2. Let $E \subset \mathbf{R}^{m}$ be a measurable set of finite measure, and let $g \in L^{1}(E)$. Given $\varepsilon>0$, we can find a positive function $\delta$ on $E$ so that

$$
\sum_{i=1}^{\infty}\left|g\left(x_{i}\right) \int_{E} \nu_{i}-\int_{E} g \nu_{i}\right|<\varepsilon
$$

for each collection $\left\{\left(\nu_{i}, x_{i}\right): i=1,2, \ldots\right\}$ such that $x_{i} \in E, \nu_{i} \geq 0$ is a measurable function on $E, S_{\nu_{i}} \subset U\left(x_{i}, \delta\left(x_{i}\right)\right), i=1,2, \ldots$, and $\sum_{i=1}^{\infty} \nu_{i} \leq 1 / \varepsilon$.

Proof. Choose an $\varepsilon>0$ and let $\eta=\varepsilon^{2} /(1+|E|)$. By the Vitali-Carathéodory theorem (see [R, Theorem 2.25]), there are extended real-valued functions $h$ and $k$ on $E$ such that $h$ is upper semicontinuous, $k$ is lower semicontinuous, $h \leq g \leq k$, and $\int_{E}(k-h)<\eta$. Find a positive function $\delta$ on $E$ so that $h(y)<g(x)+\eta$ and $k(y)>g(x)-\eta$ for each $x, y \in E$ with $|x-y|<\delta(x)$, and let $\left\{\left(\nu_{i}, x_{i}\right): i=1,2, \ldots\right\}$ be the collection from the lemma. Then

$$
\int_{E} h \nu_{i}-\eta \int_{E} \nu_{i} \leq g\left(x_{i}\right) \int_{E} \nu_{i} \leq \int_{E} k \nu_{i}+\eta \int_{E} \nu_{i},
$$

and $\int_{E} h \nu_{i} \leq \int_{E} g \nu_{i} \leq \int_{E} k \nu_{i}$ for $i=1,2, \ldots$ As $\sum_{i=1}^{\infty} \nu_{i} \leq 1 / \varepsilon$, we obtain

$$
\begin{aligned}
\sum_{i=1}^{\infty}\left|g\left(x_{i}\right) \int_{E} \nu_{i}-\int_{E} g \nu_{i}\right| & \leq \sum_{i=1}^{\infty} \int_{E}(k-h) \nu_{i}+\eta \sum_{i=1}^{\infty} \int_{E} \nu_{i} \\
& \leq \frac{1}{\varepsilon} \int_{E}(k-h)+\frac{\eta}{\varepsilon}|E|<\varepsilon .
\end{aligned}
$$

Theorem. Let $f \in L_{\mathrm{loc}}^{1}(\Omega)$ and $F(\theta)=\int_{\Omega} f \theta$ for each $\theta \in L_{\circ}^{\infty}(\Omega)$. For almost all $x \in \Omega$ the functional $F$ is weakly differentiable at $x$ and $\mathfrak{D}_{w} F(x)=f(x)$. In particular, $F$ is differentiable almost everywhere in $\Omega$ whenever $f \in L_{\mathrm{loc}}^{\infty}(\Omega)$. Proof. With no loss of generality we may assume that $|\Omega|<+\infty$ and $f \in$ $L^{1}(\Omega)$. Let $N$ be the set of all $x \in \Omega$ at which either $F$ is not weakly differentiable or $\mathfrak{D}_{w} F(x) \neq f(x)$. Given $x \in N$, there is a $\gamma(x)>0$ such that for each $\delta>0$ we can find a normalized $\theta \in B V_{+}$with $x \in S_{\theta}^{-}, d(\theta)<\delta$, $r(\theta)>\gamma(x),|\theta|_{1}<1 / \gamma(x)$, and $\left.\left.|F(\theta)-f(x)| \theta\right|_{1}|\geq \gamma(x)| \theta\right|_{1}$. Fix an integer $n \geq 1$ and set $N_{n}=\{x \in N: \gamma(x)>1 / n\}$. Choose an $\varepsilon>0$, and use Lemma 2 to find a positive function $\delta$ on $\Omega$ so that

$$
\sum_{i=1}^{\infty}\left|f\left(x_{i}\right) \int_{\Omega} \nu_{i}-\int_{\Omega} f \nu_{i}\right|<\frac{\varepsilon}{2 n}
$$

for each collection $\left\{\left(\nu_{i}, x_{i}\right): i=1,2, \ldots\right\}$ such that $x_{i} \in \Omega, \nu_{i}$ is a nonnegative measurable function on $\Omega, S_{\nu_{i}} \subset U\left(x_{i}, \delta\left(x_{i}\right)\right), i=1,2, \ldots$, and $\sum_{i=1}^{\infty} \nu_{i} \leq n$. Let $\mathscr{S}$ be the family of all closed sets $S \subset \Omega$ such that $d(S)<\bar{\delta}\left(x_{S}\right)$ for an $x_{S} \in \Omega \cap N_{n}$, and there is a measurable function $\nu_{S}$ on $\Omega$ with $0 \leq \nu_{S} \leq n, S_{\nu_{S}} \subset S, \int_{\Omega} \nu_{S} \geq|S| / 2$, and

$$
\left|f\left(x_{S}\right) \int_{\Omega} \nu_{S}-\int_{\Omega} f \nu_{S}\right| \geq \frac{1}{n} \int_{\Omega} \nu_{S} .
$$

Claim. The family $\mathscr{S}$ is a Vitali cover of $N_{n}$. 
Assuming the claim is correct, there are disjoint sets $S_{1}, S_{2}, \ldots$ in $\mathscr{S}$ such that $\left|N_{n}-\bigcup_{i=1}^{\infty} S_{i}\right|=0$ (see [S, Chapter 4, Theorem (3.1)]). If $\nu_{i}=\nu_{S_{i}}$ and $x_{i}=x_{S_{i}}, i=1,2, \ldots$, then $\sum_{i=1}^{\infty} \nu_{i} \leq n$ and hence

$$
\left|N_{n}\right| \leq \sum_{i=1}^{\infty}\left|S_{i}\right| \leq 2 \sum_{i=1}^{\infty} \int_{\Omega} \nu_{i} \leq 2 n \sum_{i=1}^{\infty}\left|f\left(x_{i}\right) \int_{\Omega} \nu_{i}-\int_{\Omega} f \nu_{i}\right|<\varepsilon .
$$

It follows from the arbitrariness of $\varepsilon$ that $\left|N_{n}\right|=0$, and as $N=\bigcup_{n=1}^{\infty} N_{n}$ we see that $|N|=0$.

Proof of the claim. Choose an $x \in N_{n}$ and a positive $\Delta \leq \delta(x)$. There is a normalized $\theta \in B V_{+}$with $x \in S_{\theta}^{-}, d(\theta)<\Delta, r(\theta)>1 / n,|\theta|_{\infty}<n$, and

$$
\left|\int_{\Omega} f \theta-f(x) \int_{\Omega} \theta\right|>\frac{1}{n} \int_{\Omega} \theta \text {. }
$$

By Lemma $1,\left|S_{\theta}\right| /\left[d\left(S_{\theta}^{1}\right]^{m} \geq 1 /(n c)^{m}\right.$ where $c>0$ is a constant depending only on $m$. Moreover, there is a compact set $K \subset S_{\theta}$ such that $|K| \geq$ $\left|S_{\theta}\right| / 2, \int_{K} \theta \geq \frac{1}{2} \int_{\Omega} \theta=\left|S_{\theta}\right| / 2$, and

$$
\left|\int_{K} f \theta-f(x) \int_{K} \theta\right| \geq \frac{1}{n} \int_{K} \theta
$$

Since

$$
\frac{|K \cup\{x\}|}{[d(K \cup\{x\})]^{m}} \geq \frac{|K|}{\left[d\left(S_{\theta}\right)\right]^{m}} \geq \frac{1}{2(n c)^{m}},
$$

it suffices to let $S=K \cup\{x\}, x_{S}=x$, and set $\nu_{S}(y)=\theta(y)$ if $y \in S$ and $\nu_{S}(y)=0$ if $y \in \Omega-S$.

Example. Let $m=2, \Omega=\mathbf{R}^{2}$, and let $\left\{x_{1}, x_{2}, \ldots\right\}$ be an enumeration of a countable dense set $D \subset \Omega$. The characteristic function of $E \subset \Omega$ is denoted by $\chi_{E}$. We define an $f \in L^{1}(\Omega)$ by setting

$$
f(x)=\sum_{n=1}^{\infty}\left|x-x_{n}\right|^{-3 / 2} \chi_{U\left(x_{n}, 2^{-n}\right)}(x)
$$

for each $x \in \Omega-D$. Clearly, $f$ is essentially bounded in no nonempty open subset of $\Omega$, and we show that the functional $F: \theta \mapsto \int_{\Omega} f \theta$ on $L_{\circ}^{\infty}(\Omega)$ is nowhere differentiable. To this end select a $z \in \Omega$, and let $\left\{k_{n}\right\}$ be an increasing sequence of positive integers such that $U\left(x_{k_{n}}, 2^{-2 k_{n}}\right) \subset U\left(z, 2^{-n}\right)$ for $n=1,2, \ldots$. If

$$
\theta_{n}=\chi_{U\left(z, 2^{-n}\right)}+2^{k_{n}} \chi_{U\left(x_{k_{n}}, 2^{-2 k_{n}}\right)},
$$

then $\theta_{n} \in B V_{+},\left\|\theta_{n}\right\| \leq \pi 2^{2-n}$, and $\pi 2^{-2 n} \leq\left|\theta_{n}\right|_{1} \leq \pi 2^{1-2 n}$. Since

$$
F\left(\theta_{n}\right) \geq 2^{k_{n}} \int_{U\left(x_{k_{n}}, 2^{\left.-2 k_{n}\right)}\right.}\left|x-x_{k_{n}}\right|^{-3 / 2} d x=4 \pi,
$$

$x \in S_{\theta_{n}}, d\left(\theta_{n}\right)=2^{1-n}$, and $r\left(\theta_{n}\right) \geq 1 / 4$ for $n=1,2, \ldots$, we see that $F$ is not differentiable at $z$.

\section{ACKNOWLEDGMENT}

The author is obliged to Pertti Mattila for valuable discussions concerning functions of bounded variation. 


\section{REFERENCES}

[HN] E. P. Hamilton and M. Z. Nashed, Global and local variational derivatives and integral representations of Gâteaux differentials, J. Funct. Anal. 49 (1982), 128-144.

[G] E. Giusti, Minimal surfaces and functions of bounded variation, Birkhäuser, Basel, 1984.

[KMP] J. Kurzweil, J. Mawhin, and W. F. Pfeffer, An integral defined by approximating BV partitions of unity, Czechoslovak Math. J. (to appear).

[P1] W. F. Pfeffer, On the continuity of the Volterra variational derivative, J. Funct. Anal. 71 (1987), 195-197.

[R] W. Rudin, Real and complex analysis, McGraw-Hill, New York, 1987.

[S] S. Saks, Theory of the integral, Dover, New York, 1964.

Department of Mathematics, University of California, Davis, California 95616

E-mail address: wfpfeffer@ucdavis.edu or wfpfeffer@ucdavis.bitnet 